\title{
Genome-wide RNAi analysis of JAK/STAT signaling components in Drosophila
}

\author{
Gyeong-Hun Baeg, ${ }^{1,2}$ Rui Zhou, ${ }^{1,2}$ and Norbert Perrimon ${ }^{1,3}$ \\ ${ }^{1}$ Department of Genetics, Howard Hughes Medical Institute, Harvard Medical School, Boston, MA 02115, USA
}

The cytokine-activated Janus kinase (JAK)/signal transducer and activator of transcription (STAT) pathway plays an important role in the control of a wide variety of biological processes. When misregulated, JAK/STAT signaling is associated with various human diseases, such as immune disorders and tumorigenesis. To gain insights into the mechanisms by which JAK/STAT signaling participates in these diverse biological responses, we carried out a genome-wide RNA interference (RNAi) screen in cultured Drosophila cells. We identified 121 genes whose double-stranded RNA (dsRNA)-mediated knockdowns affected STAT92E activity. Of the 29 positive regulators, 13 are required for the tyrosine phosphorylation of STAT92E. Furthermore, we found that the Drosophila homologs of RanBP3 and RanBP10 are negative regulators of JAK/STAT signaling through their control of nucleocytoplasmic transport of STAT92E. In addition, we identified a key negative regulator of Drosophila JAK/STAT signaling, protein tyrosine phosphatase PTP61F, and showed that it is a transcriptional target of JAK/STAT signaling, thus revealing a novel negative feedback loop. Our study has uncovered many uncharacterized genes required for different steps of the JAK/STAT signaling pathway.

[Keywords: JAK/STAT signal transduction pathway; Drosophila; RNA interference]

Supplemental material is available at http://genesdev.org.

Received April 1, 2005; revised version accepted June 20, 2005.

The evolutionarily conserved Janus kinase (JAK)/signal transducer and activator of transcription (STAT) cascade plays a key role in a wide variety of biological processes such as the immune response, tumorigenesis, and development. This pathway, regulated by a large number of cytokines and growth factors, has emerged as an essential facet of vertebrate signaling. Critical roles of the receptor-associated JAKs and their substrate transcription factors STATs have been demonstrated through the generation of gene knockout mice. JAK1-deficient mice die perinatally and are unable to nurse (Rodig et al. 1998), while JAK2 mutant mice display embryonic lethality due to anemia (Neubauer et al. 1998; Parganas et al. 1998). Mice lacking JAK3 have profound reductions in thymocytes, B and T cells, as observed in the case of autosomal severe combined immune deficiency (SCID) mice (Nosaka et al. 1995; Park et al. 1995; Thomis et al. 1995). Similarly, STAT-deficient mice are either immunocompromised or display hematopoietic defects (Durbin et al. 1996; Meraz et al. 1996). On the other hand, constitutive activation of JAKs and/or STATs is correlated with tumorigenesis through their intimate

\footnotetext{
${ }^{2}$ These authors contributed equally to this work.

${ }^{3}$ Corresponding author.

E-MAIL perrimon@receptor.med.harvard.edu; FAX (617) 432-7688.

Article published online ahead of print. Article and publication date are

at http://www.genesdev.org/cgi/doi/10.1101/gad.1320705.
}

connection to growth factor signaling, apoptosis, and angiogenesis (Yu and Jove 2004). Furthermore, studies in model genetic systems, such as Drosophila, Xenopus, and zebrafish have shown that the JAK/STAT pathway participates in an unusually broad set of developmental decisions that include cell fate determination, cell migration, planar cell polarity, convergent extension, and stem cell maintenance (Hou et al. 2002). Although much work has been done on this pathway, many questions remain to be addressed. In particular, the exact molecular mechanisms by which JAK/STAT signaling integrates and transduces cues from numerous extracellular signaling molecules to trigger specific genetic programs in vivo remain to be elucidated. In addition, STATs have been shown to be activated by at least four distinct mechanisms in mammals (Bromberg 2001), and many aspects of this regulation remain only partially understood.

In mammals, genetic approaches to identify and characterize components of the JAK/STAT pathway have been predominantly dependent on cell-line-based genetics or gene targeting, which is labor-intensive and often time-consuming (Velazquez et al. 1992). Moreover, interpretation of mammalian genetic studies is further complicated by the redundancy within individual components of the JAK/STAT pathway. In contrast, Drosophila melanogaster is highly amenable to genetic manipulations and has served as an excellent model organ- 
ism to study the JAK/STAT pathway. Genetic studies in Drosophila have identified several canonical components of the JAK/STAT pathway, including cytokinelike molecules Unpaired (Upd); Domeless/Master of Marelle (Dome/Mom), the Upd receptor distantly related to the mammalian gp130 subfamily; Hopscotch (Hop), the Drosophila homolog of vertebrate JAK; STAT92E, the STAT protein; and SOCS36E, a negative regulator of the JAK/STAT pathway (Hou et al. 2002). However, the inherent limitations of forward genetic approaches make it likely that many genes remain unidentified. Recently, the development of high-throughput genome-wide RNAi-based technology in cultured Drosophila cells offers a rapid, systematic, and complementary methodology for dissecting gene functions (Boutros et al. 2004; Dasgupta et al. 2005). This quantitative cell-based RNAi analysis also offers the advantage of uncovering gene function associated with subtle phenotypes and/or redundancy that might not be readily identifiable through genetic studies, including those in sensitized genetic backgrounds (Bach et al. 2003). Furthermore, with abundant genetic tools readily available, Drosophila is a superior genetically tractable system for the in vivo validation of candidate genes.

There are a number of steps involved in signaling through the JAK/STAT pathway, including phosphorylation and nucleocytoplasmic shuttling of STAT92E. We hoped to identify new members of this canonical pathway as well as proteins that might function as modulators by regulating different steps of this pathway. To this end, we performed a genome-wide RNAi screen in cultured Drosophila cells and identified 121 genes that represent 29 potential positive and 92 negative regulators of the JAK/STAT pathway. Importantly, among these were five canonical components of the JAK/STAT pathway, including Upd2, Dome, Hop, STAT92E, and SOCS36E, indicating the robustness and validity of this approach. The 29 positive regulators were further analyzed by examining the effect of their double-stranded RNA (dsRNA)-mediated knockdown on STAT92E tyrosine phosphorylation. We also demonstrate that Drosophila homologs of RanBP3 and RanBP10 are involved in STAT92E nucleocytoplasmic transport. Finally, we characterized the first protein tyrosine phosphatase, PTP61F, that negatively regulates the Drosophila JAK/STAT pathway. Together, these findings underscore the robustness of genome-wide RNAi screening approaches to uncover novel regulators involved in different steps in signaling pathways.

\section{Results}

\section{Generating a JAK/STAT reporter gene in Drosophila}

SOCS36E (Drosophila homolog of suppressor of cytokine signaling gene family) encodes a negative regulator of the JAK/STAT signaling pathway in Drosophila, and has been shown to be transcriptionally activated by JAK/STAT signaling (Callus and Mathey-Prevot 2002; Karsten et al. 2002). Upon close examination of the SOCS36E genomic region, we identified a 441-bp frag- ment in the enhancer of the SOCS36E gene that contains two potential STAT92E-binding sites. To generate a JAK/STAT reporter, we placed five tandem repeats of this genomic fragment upstream of a minimal heatshock promoter-driven cDNA encoding the firefly luciferase gene (Fig. 1A), herein referred to as 10XSTAT92Eluciferase. To confirm that this reporter gene was responsive to JAK/STAT signaling and to select a Drosophila cell line that would allow for the identification of both positive and negative regulators of STAT92E activity, we first transfected various Drosophila cell lines with 10XSTAT92E-luciferase and an Actin promoter-driven Renilla luciferase expression vector (Act-Renilla) together with various dsRNAs. We tested dsRNAs against known JAK/STAT components and quantified the activity of JAK/STAT signaling by measuring relative luciferase units (RLU), which equaled the ratio of the absolute activity of firefly luciferase to Renilla luciferase. A Drosophila Schneider cell line derivative (S2-NP) exhibited robust endogenous JAK/STAT activity, and this activity was sensitive to RNAi manipulations. The addition of dsRNAs against positive regulators, such as STAT92E, Hop, and Dome, led to a 12- to 24-fold decrease in the reporter activity, whereas dsRNA against a negative regulator, SOCS36E, increased its activity by threefold (Fig. 1B). Thus the reporter gene faithfully reflected JAK/STAT signaling in S2-NP cells.

\section{A cell-based genome-wide RNAi screen and data analysis}

To identify additional modulators of the JAK/STAT pathway whose loss-of-function affects STAT92E activ-

\section{A}

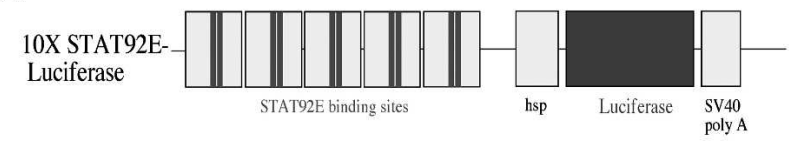

B

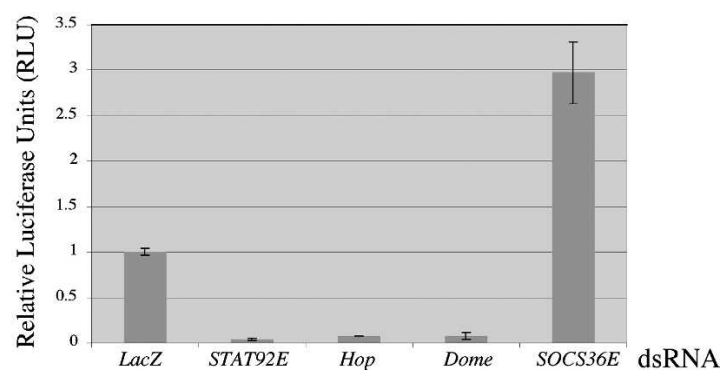

Figure 1. Generating a JAK/STAT reporter construct. $(A)$ Schematic representation of the 10XSTAT92E-luciferase reporter construct. Five copies of a genomic fragment from the SOCS36E intronic region containing two STAT92E-binding sites were placed upstream of a hsp minimal promoter-driven firefly luciferase gene. (B) Drosophila S2-NP cells were transfected with 10XSTAT92E-luciferase and Act-Renilla together with dsRNAs against various canonical components of the JAK/ STAT pathway. Luciferase assay was performed $4 \mathrm{~d}$ later, and the reporter activity was normalized as the ratio of firefly/Renilla. Note that the control value was set to 1 . The results were from two independent experiments. 
ity, we performed a genome-wide RNAi screen using cultured Drosophila S2-NP cells in 384-well plates. We used a library of $\sim 21,300$ dsRNAs (Boutros et al. 2004) that target $>95 \%$ of the annotated genes in the Drosophila genome (Hild et al. 2003). Luciferase values were analyzed and potential candidate genes were assigned on the basis of their deviation from the plate average for each given plate (see Materials and methods). In the primary screen, we identified 474 candidate genes that include 259 genes that reduced JAK/STAT signaling by more than two standard deviations (SD) and 215 genes that increased signaling by more than three SD when knocked down by RNAi (Fig. 2A). Importantly, among these genes we independently identified five canonical components of the JAK/STAT pathway: Upd2, Dome, Hop, STAT92E, and SOCS36E, confirming the robustness of the screen (Fig. 2B).

Of these 474 candidate genes, 188 represent sequences not annotated by the Berkeley Drosophila Genome Project (which were based on an inclusive algorithm for determining ORFs in the fly genome) (Hild et al. 2003), ribosomal proteins, and proteins involved in RNA processing and translation (data not shown). These genes were not pursued further. We next repeated the same assay with the remaining 286 genes in duplicate. Twohundred-two candidate genes $(71 \%)$ identified in the primary screen were verified. In the primary screen, we used Act-Renilla for normalizing the transfection efficiency. Because the candidate genes from the primary screen were determined by calculating the Firefly/Renilla ratio, it is conceivable that some of them might arise from the effect of certain dsRNAs on the Actin promoter. Thus, to remove candidate genes that might affect the Actin promoter and not the JAK/STAT responsive element, we repeated our reporter assay using a Pol III promoter-driven Renilla luciferase expression vector (pol III-Renilla). We found that 81 candidates $(40 \%)$ fell into this category and were not pursued further (data not shown). Thus this screen identified 121 candidate genes that specifically modulate JAK/STAT signaling in S2-NP cells (Supplementary Table 1). Importantly, Upd2, a cytokine-like molecule, is among the positive regulators identified in the screen. Thus, the endogenously expressed Upd2 is responsible for basal levels of JAK/STAT signaling in S2-NP cells.

These 121 genes were assigned to categories based on their predicted molecular functions, protein domains, and reports from the literature to help us to predict functions and generate testable hypotheses for further characterization (Fig. 2B). These categories include (1) canonical JAK/STAT signaling pathway component, (2) kinase/phosphatase, (3) chromatin remodeling, (4) protein trafficking, (5) cell adhesion, (6) structural molecule, (7) transcription factor, and (8) miscellaneous.

Next, we assayed the 29 positive regulators in cells stimulated with exogenous Upd, a well-characterized ligand for JAK/STAT signaling. We found that 27 genes were validated in this assay, strongly suggesting that the screen has identified bona fide components of the JAK/ STAT signaling pathway (Supplementary Table 1). This assay revealed that $\mathrm{Upd} 2$ and CG17836 are not required for Upd-induced JAK/STAT signaling. Since Upd2 is an endogenously expressed cytokine responsible for basal levels of JAK/STAT signaling, it is not expected to be required for the JAK/STAT signal elicited by exogenous Upd.

\section{Identification of genes that affect tyrosine phosphorylation of STAT92E}

To more clearly elucidate the roles of positive regulators, we assayed their requirement for the phosphorylation of STAT92E. Tyrosine phosphorylation is a key step in STAT activation upon cytokine/receptor stimulation.

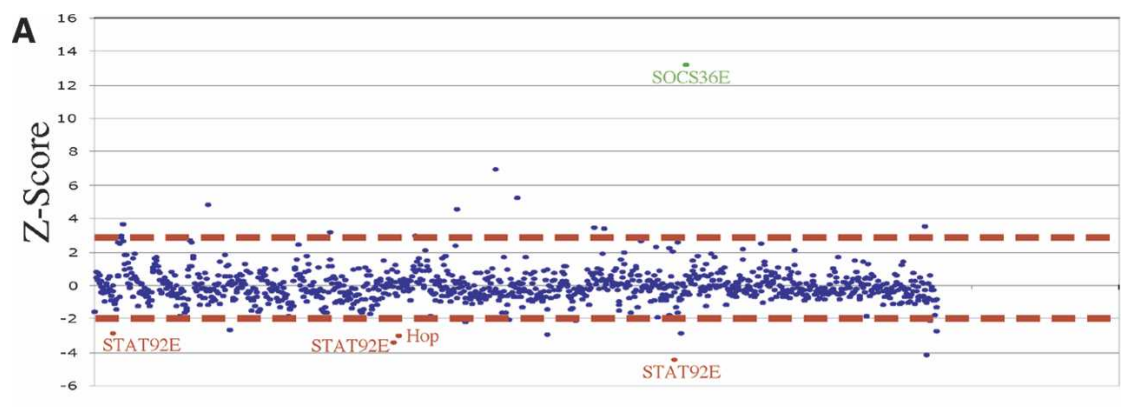

B

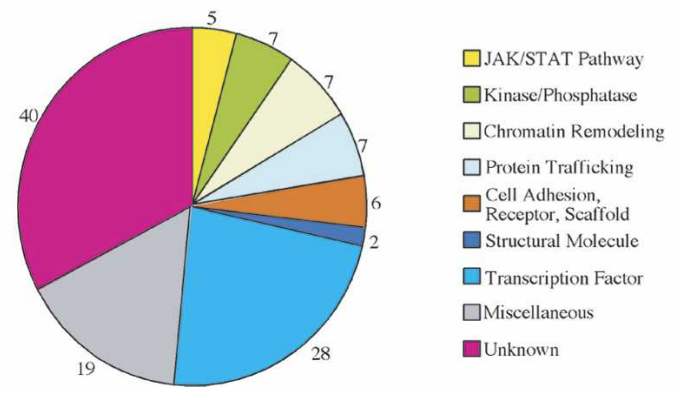

Figure 2. Data analysis for the JAK/STAT screen. (A) Scatter plot for three representative screen plates. Cutoffs were set as $2 \mathrm{SD}$ below the mean or $3 \mathrm{SD}$ above the mean RLU. Note that all three "spiked in" control dsRNAs against STAT92E were identified. (B) Pie chart depicting categories of genes identified in the JAK/STAT screen. 
Thus, monitoring steady-state levels of phosphorylated STAT in dsRNA-treated cells would provide insight into the molecular functions of our candidate genes. As expected, we found that Upd stimulation of S2-NP cells leads to a dramatic increase in tyrosine-phosphorylated STAT92E, as shown by Western blot analysis (Fig. 3A). Next, we tested the effect of dsRNAs against the 29 positive regulators on Upd-induced STAT92E phosphorylation. Thirteen genes (besides STAT92E) were found to be required for Upd-induced STAT92E phosphorylation (Fig. 3B,C; Supplementary Table 1). As expected, these genes included the canonical components Dome and hop. In contrast to the initial assay in the primary screen, here we used exogenous Upd to activate STAT92E phosphorylation, and thus we were unable to identify genes that act upstream of the receptor, such as Upd2. Notably, two of the 13 genes (CG16790 and CG4329) that regulate STAT92E phosphorylation have no predicted function, yet clearly have human orthologs; further investigation of their molecular functions in JAK/STAT signaling in Drosophila may advance our understanding of the mammalian pathway.

Interestingly, this assay revealed that RNAi knockdown of the cyclin-dependent kinase 2 gene (cdc2) resulted in a decrease in STAT92E tyrosine phosphorylation (Fig. 3B), suggesting that cdc2 modulates JAK/STAT signaling by affecting tyrosine phosphorylation of STAT92E. Consistent with this observation, Warts/Lats,

A

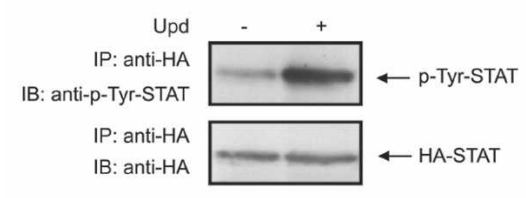

B

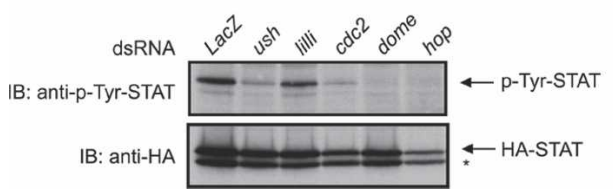

Figure 3. Identification of genes required for Upd-induced tyrosine phosphorylation of STAT92E. (A) Act-STAT92E-HA was transfected into S2-NP cells together with dsRNA against lacZ. Cells were split into two dishes $3.5 \mathrm{~d}$ after transfection. Half of the cells were cocultured with S2-NP cells transfected with Act-Upd $\sim 12 \mathrm{~h}$ prior to harvest and the other half remained untreated. Cell extracts were subjected to immunoprecipitation using anti-HA antibodies and the immunoprecipitates were analyzed by immunoblotting using anti-phospho-Tyr-STAT92E and HA antibodies. Note that Upd-induction leads to a dramatic increase in STAT92E phosphorylation. (B) Act-STAT92E-HA was transfected into S2-NP cells together with various dsRNAs targeting positive regulators identified in the screen. Cells were stimulated with Upd $\sim 12 \mathrm{~h}$ prior to harvest. Cell extracts were subjected to Western blot analysis using anti-phospho-TyrSTAT92E and HA antibodies. dsRNAs against LacZ and lilli serve as control. $(C)$ List of genes required for Upd-induced STAT92E phosphorylation. which has been shown both biochemically and genetically to interact with cdc2 and to negatively regulate its kinase activity (Tao et al. 1999), was identified in our screen as a potential negative regulator of JAK/STAT signaling. These results suggest that STAT92E plays an important role in Warts/Lats-mediated inhibition of cell proliferation.

We also identified echinoid (ed) as a positive regulator required for Upd-dependent STAT92E tyrosine phosphorylation. ed encodes a cell adhesion molecule and has been shown to be a negative regulator of the EGFR signaling pathway during Drosophila eye development (Bai et al. 2001; Islam et al. 2003). Previous experiments have shown both positive and negative interactions between the JAK/STAT pathway and the EGFR pathway. For example, STAT92E mutants phenocopy mutants in the EGFR pathway (Yan et al. 1996). Furthermore, studies using mammalian tissue culture systems have demonstrated that EGFR signaling activates both JAK1 and STAT1 (Quelle et al. 1995; Leaman et al. 1996). In addition, EGFR-induced cell migration is mediated predominantly by the JAK/STAT pathway in primary esophageal keratinocytes (Andl et al. 2004). Similarly, ed has been shown to be responsible for defective cell migration in Caenorhabditis elegans (Vogel and Hedgecock 2001). Therefore studying the role of ed in JAK/STAT signaling in different contexts may facilitate our understanding of the genetic and biochemical mode of STAT activation by EGFR signaling, and provide insights into the mechanisms governing cancer cell metastasis in humans.

\section{Identification of genes that affect nuclear translocation of STAT92E}

Another step in the activation of the JAK/STAT signaling pathway is the translocation of STATs into the nucleus. In resting cells, STATs reside mainly in the cytoplasm. Upon cytokine stimulation, they are phosphorylated on key tyrosine residues and rapidly translocate to the nucleus, where they trans-activate target genes. Previous studies have shown that Importin $\alpha 5$ and Ran are required for the nuclear import of phosphorylated (activated) STATs (Sekimoto et al. 1997). To reset the cells after stimulation, STATs are exported out of the nucleus into the cytoplasm in preparation for the next round of signaling using an Exportin-1/CRM-1-dependent mechanism (McBride et al. 2000). These observations suggest that defective nucleocytoplasmic shuttling of STATs can disrupt steady-state distribution of STATs and induce aberrant biological responses. Among all 121 candidates, we identified seven genes that are potentially involved in protein trafficking based on their predicted molecular functions and protein domains (Fig. 2B; Supplementary Table 1). These include Rab26, Ran, CG10225, which encodes the Drosophila homolog of Ran-binding protein 3 (RanBP3), CG11763, which encodes the Drosophila homolog of Ran-binding protein 10 (RanBP10), and the Drosophila homolog of Cellular Apoptosis Susceptibility gene product (CAS) that was initially identified as a Ran-binding protein. In addition, we 
also identified Drosophila homologs of Transportin 1 and Nucleoporin 196, which have been implicated in protein import and/or export in mammals. We therefore examined the subcellular localization of phosphorylated STAT92E under conditions where each of the seven candidates was depleted by RNAi except Rad26. As a control we found that under resting conditions tyrosine phosphorylated STAT92E was detected predominantly in the cytoplasm (Fig. 4A-C). Moreover, we observed a significant reduction in phosphorylated STAT92E levels in the cytoplasm when cells were treated with dsRNA against

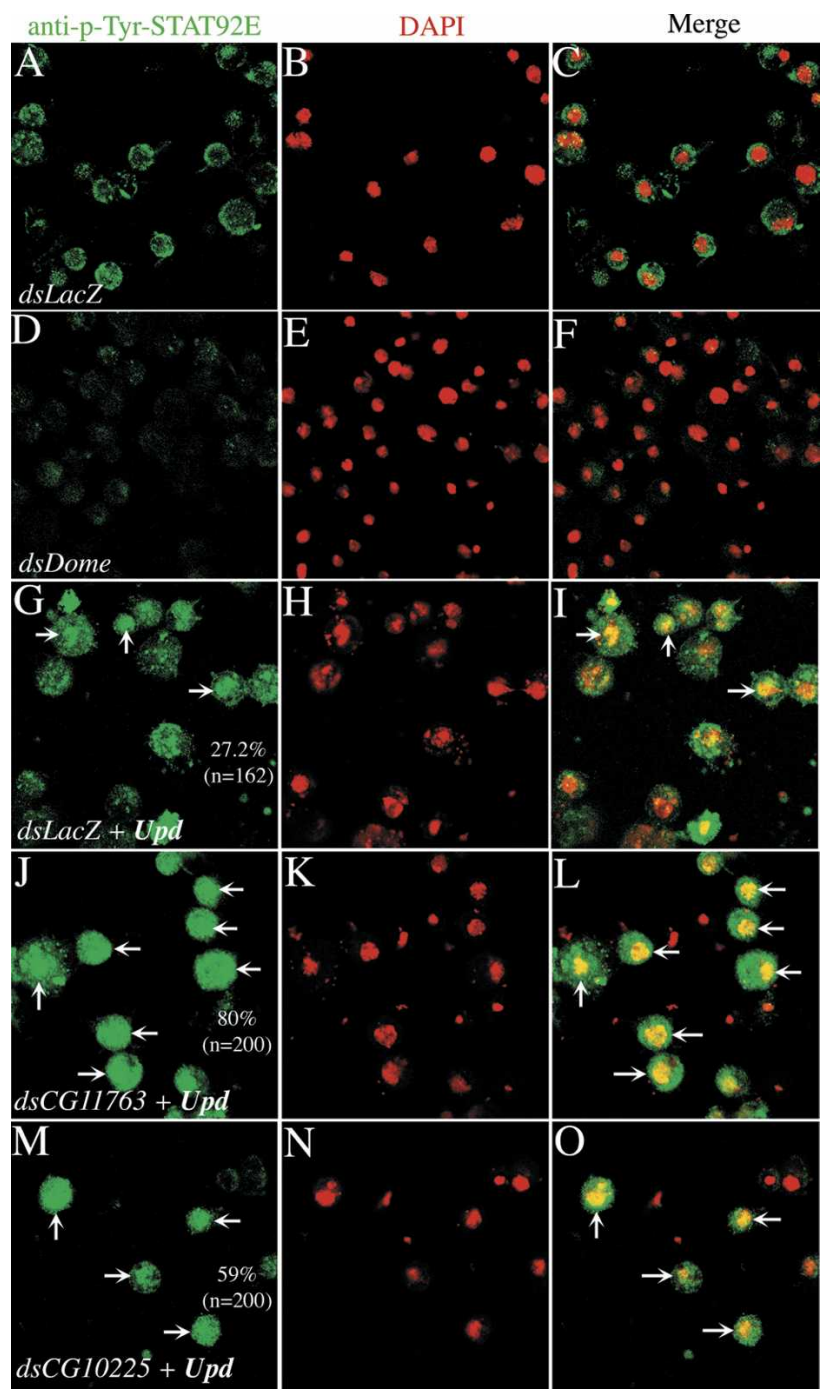

Figure 4. Drosophila homologs of RanBP3 (CG11763) and RanBP10 (CG10225) are involved in phosphorylated STAT92E nucleocytoplasmic shuttling. Cells were treated with various dsRNAs and then transfected with Act-Upd $4 \mathrm{~d}$ later $(G-O)$ or remained untransfected $(A-F)$. Immunostaining was performed using anti-phospho-Tyr-STAT92E antibody (green). DAPI staining was employed to visualize the nuclei (red). Note that a significant accumulation of phosphorylated STAT92E in the nuclei of cells treated with dsRNA against either CG11763 or CG10225 was detected upon Upd induction, compared with cells treated with dsRNA for lacZ. the receptor dome (Fig. 4D-F). Upon stimulation with Upd, STAT92E accumulates in the nuclei of $27.2 \%$ $(n=162)$ of cells (Fig. 4, cf. G-I and A-C). These results illustrate the specificity and sensitivity of our assay. Interestingly, we found that cells treated with dsRNAs against CG11763 or CG10225 displayed a significant increase in phospho-STAT92E nuclear accumulation upon Upd stimulation (Fig. 4, cf. J-L,M-O and G-I, 80\%, $n=200$ and $59 \%, n=200$, respectively). This was not due to changes in the total phosphorylation levels of STAT92E (data not shown). We could not detect significant effects of dsRNA-mediated knockdown of Cas or Trn on STAT92E translocation (data not shown). On the other hand, the role of Ran and Nup98 in STAT92E translocation could not be assessed in this assay due to difficulties in introducing the Upd expression vector into cells upon RNAi knockdown of these two genes (data not shown). Taken together, these results strongly suggest that the Drosophila homologs of RanBP3 and RanBP10 are novel regulators of JAK/STAT signaling that affect signal-dependent STAT92E nuclear transport.

The role of protein tyrosine phosphatase 61F (PTP61F) in the IAK/STAT pathway

Another important step in the JAK/STAT signal transduction pathway is the dephosphorylation of the signaling molecules JAKs and STATs. In mammals, several PTPs have been implicated in the dephosphorylation of JAK and/or STAT proteins both in the cytoplasm and in the nucleus (Shuai and Liu 2003). In contrast, no PTPs have been identified that regulate JAK/STAT signaling in Drosophila. PTP61F was identified as a strong negative regulator in our screen. Knockdown of PTP61F by RNAi resulted in a more than fourfold increase in STAT92E-dependent reporter activity (Fig. 5A). PTP61F encodes the Drosophila homolog of mammalian PTP-1B, which has been shown to attenuate insulin, PDGF, EGF, and IGF-I signaling by dephosphorylating tyrosine residues of JAKs and/or STATs in mammalian tissue culture (Aoki and Matsuda 2000; Myers et al. 2001). We therefore tested the hypothesis that PTP61F might serve as the tyrosine phosphatase for Hop. We observed a dramatic increase in tyrosine phosphorylation of Hop upon RNAi knockdown of PTP61F (Fig. 5B), suggesting that Hop may be a substrate of PTP61F. We also detected a significant increase in STAT92E phosphorylation in cells treated with dsRNA against PTP61F (Fig. 5C). This is consistent with the notion that STAT92E is a downstream target of Hop, although we cannot rule out the possibility that both Hop and STAT92E may be targets of PTP61F.

In both mammals and Drosophila, SOCS, a negative regulator of the JAK/STAT pathway, has been shown to be transcriptionally activated by JAK/STAT signaling, thus generating a negative feedback loop. This prompted us to examine the expression pattern of PTP61F and whether its expression is responsive to JAK/STAT signaling in vivo. We found PTP61F is expressed in a striped pattern, reminiscent of the STAT92E expression pattern 
Baeg et al.

A

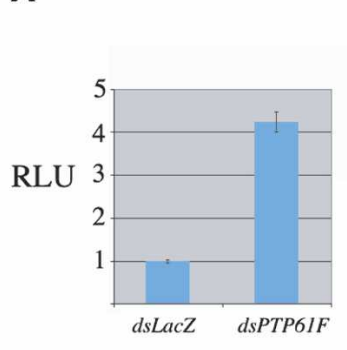

B

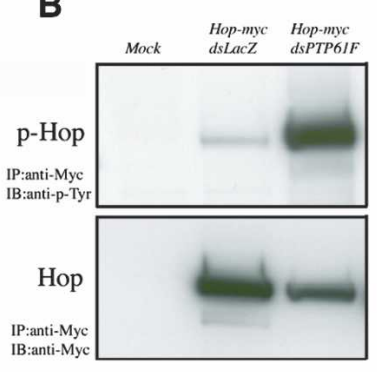

C

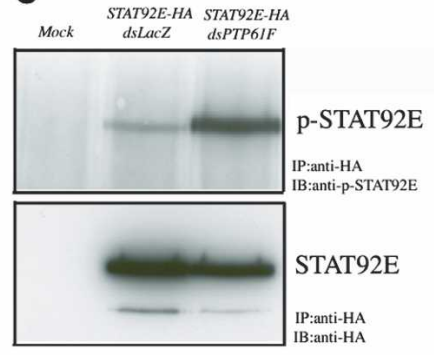

D

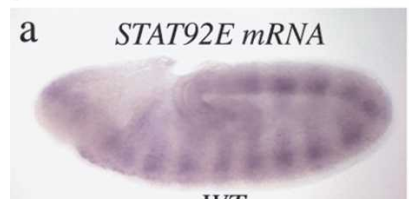

$W T$

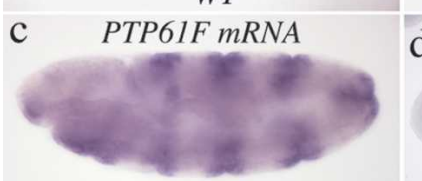

UAS-Upd/+;prd-Gal4/+

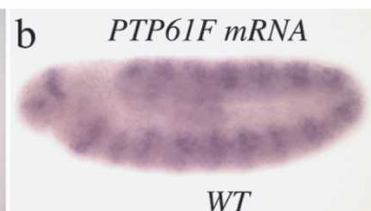

d PTP61F $m R N A$

E

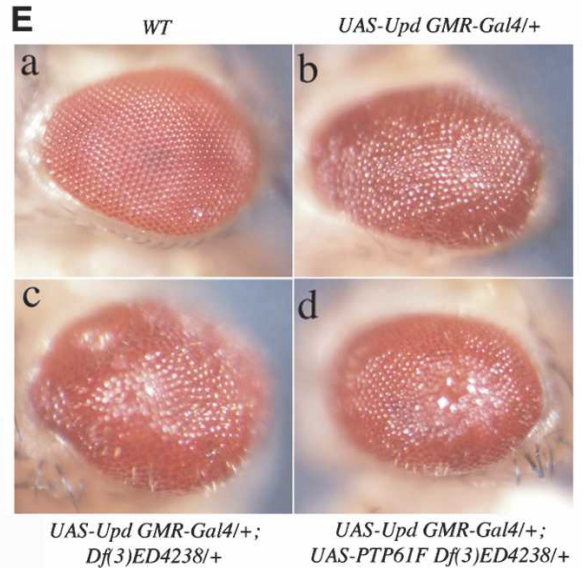

Figure 5. PTP61F negatively regulates the JAK/STAT pathway in Drosophila. (A) Knockdown of PTP61F by RNAi activates the JAK/STAT reporter activity. Drosophila S2-NP cells were transfected with 10XSTAT92E-luciferase and Act-Renilla together with dsRNAs against lacZ or PTP61F. Luciferase assay was performed $4 \mathrm{~d}$ later and the reporter activity was normalized as the ratio of firefly to Renilla. The control value was set as 1 . The results were from two independent experiments. (B) Act-Myc-Hop was transfected into S2-NP cells together with dsRNAs against lacZ or PTP61F. Cells were harvested and cell lysates were immunoprecipitated with anti-Myc antibody. Immunoprecipitates were analyzed by immunoblotting using anti-phospho-Tyr or anti-Myc antibodies. Note that an increase in phospho-Hop levels was detected upon RNAi knockdown of PTP61F. (C) Act-STAT92E-HA was transfected into S2-NP cells together with dsRNAs against lacZ or PTP61F. Cells were harvested and cell lysates were immunoprecipitated with anti-HA antibody. Immunoprecipitates were analyzed by immunoblotting using anti-phospho-Tyr-STAT92E or anti-HA antibodies. An increase in phospho-STAT92E levels was detected upon RNAi knockdown of PTP61F. (D) RNA in situ hybridization using STAT92E or PTP61F probes was performed on wild-type stage 9-10 embryos (panels $a, b$ ), embryos overexpressing Upd under the control of paired-Gal4 (panel c), and hop GLC embryos (panel d). Note that PTP61F transcript levels are dramatically increased in the paired domain (panel $c$ ) and decreased in embryo lacking hop activity (panel $d$ ). (E) Genetic interactions between Upd and PTP61F. Overexpression of $U p d$ in the eye under the control of GMR-Gal4 results in a dramatic overgrowth and deformation in the adult eye (cf. panels $a$ and $b$ ). Removing one copy of PTP61F further enhances this phenotype (panel $c$ ), whereas introduction of a PTP61F transgene rescues this phenotype (panel $d$ ).

(Fig. 5D, panels a,b). In addition, overexpression of Upd under the control of prd-Gal4 resulted in a dramatic increase in PTP61F transcript levels in the paired domain (Fig. 5D, panel c). Furthermore, levels of the PTP61F transcript were greatly reduced in embryos lacking Hop activity (Fig. 5D, panel d), suggesting that PTP61F transcription is dependent on active JAK/STAT signaling. Taken together, these results demonstrate that PTP61F expression responds to JAK/STAT signaling in vivo.

These data suggested that loss of PTP61F would result in an increase in JAK/STAT signaling. Thus, we next examined the genetic interaction between PTP61F and canonical components of the JAK/STAT pathway, using $D f(3) E D 4238$, a deficiency uncovering the PTP61F gene. We tested the interaction in the Drosophila eye following overexpression of Upd using GMR-Gal4 driver, which causes a dramatic overgrowth and deformation of the adult eye (Fig. 5E, panel b) (Bach et al. 2003; Chen et al. 2003). The severity of this phenotype is proportional to the strength of the JAK/STAT-mediated signal, as removing one copy of STAT92E significantly suppresses the GMR-Upd eye phenotype (Bach et al. 2003; data not shown). Consistent with PTP61F being a negative regulator of the JAK/STAT signaling pathway, flies heterozygous for $D f(3) E D 4238$ showed an enhanced deformed eye phenotype (Fig. 5E, panel c). A PTP61F transgene rescues this enhanced deformed eye phenotype in flies heterozygous for $D f(3) E D 4238$ (Fig. 5E, panel d). In addition, the PTP61F transgene also rescues lethality in flies carrying UAS-Upd GMR-Gal4/+; Df(3)ED4238/+, presumably caused by leaky expression of $U A S-U p d$ in conjunction with PTP61F deficiency (Supplementary Table 2).

Next we examined the genetic interaction between 
PTP61F and Hop. Flies carrying a dominant hyperactive Hop allele $\left(\mathrm{Hop}^{\text {Tum-1 }}\right)$ display decreased viability and the formation of melanotic tumors (Harrison et al. 1995; Dearolf 1998). This tumor formation phenotype is sensitive to gene dosage. Previous studies have shown that reducing the levels of positive regulators, such as STAT92E, Cdk4, and CycE, increases the viability and/ or decreases tumor formation (Hou et al. 1996; Chen et al. 2003). We therefore monitored both viability and melanotic tumor formation in females heterozygous for Hop ${ }^{\text {Tum-l }}$ and compared these results to females heterozygous for both Hop ${ }^{\text {Tum-1 }}$ and $D f(3) E D 4238$. Removing one copy of PTP61F in Hop ${ }^{\text {Tum-l }}$ heterozygous females leads to a significant decrease in survival rate and a dramatic enhancement in the formation of melanotic tumors (Table 1). Altogether, these results demonstrate that PTP61F is a bona fide negative regulator of the JAK/ STAT pathway in Drosophila.

\section{Discussion}

Here, we report the first genome-wide RNAi screen for novel components of the JAK/STAT signal transduction pathway. This screen has uncovered 116 novel genes that regulate JAK/STAT signaling in Drosophila, in addition to five previously characterized canonical JAK/ STAT components. This demonstrates that our screen was successful in identifying genes with specific roles in the JAK/STAT pathway. We further showed that 13 of the 29 positive regulators are required for Upd-induced STAT92E phosphorylation. In addition, we found two novel regulators of STAT92E nuclear translocation. Finally, we identified PTP61F as the first protein tyrosine phosphatase that negatively regulates JAK/STAT signaling in Drosophila both in vitro and in vivo, and demonstrated that PTP61F is a transcriptional target of JAK/ STAT signaling.

Among the identified genes, $40(32.8 \%)$ had no predicted molecular function and/or recognizable protein domain, suggesting that the screen identified many uncharacterized genes with essential roles in JAK/STAT signaling (Fig. 2B; Supplementary Table 1). Notably, "Reciprocal-Best-Blast" (RBB) analysis revealed that 88 genes $(72.7 \%)$ identified in the screen have human orthologs, suggesting a conserved role in the mammalian JAK/STAT signaling pathway (Supplementary Table 1).

The list of candidate genes identified in this screen

Table 1. Loss of function of PTP61F enhances Tum-1 phenotypes

\begin{tabular}{lcc}
\hline Genotype & Viable & \% \\
\hline Tum-1/+; TM3, Sb/+ & 350 & 19.43 \\
Tum-1/+; Df(3)ED4238/+ & 177 & 89.83 \\
\hline
\end{tabular}

Results from three independent experiments.

Female flies heterozygous for both $D f(3) E D 4238$ and hop Tum-1 displayed a significant decrease in viability and a dramatic increase in melanotic tumor formation compared to hop ${ }^{\text {Tum-l }}$ female flies heterozygous for only hop ${ }^{\text {Tum-1 }}$. only minimally overlaps with that generated from similar genome-wide RNAi studies on the Wnt and Hedgehog signaling pathways (Dasgupta et al. 2005; K. Nybakken, pers. comm.), indicating that we have identified many genes that have a specific role in the JAK/STAT signaling pathway. Moreover, $\sim 73 \%$ of our candidate genes have well-conserved human orthologs, suggesting that cell-based assays in Drosophila can serve as a simple assay system to rapidly identify and characterize genes that may play similar roles in the mammalian JAK/ STAT pathway.

Clearly the results from the screen presented here will provide the foundation for many follow-up investigations, as each of the newly identified genes will need to be carefully validated in vivo for their roles in JAK/STAT signaling. Validation in the fly system, as well as in other model systems for those evolutionarily conserved factors, will provide further insights into our global understanding of JAK/STAT signaling.

\section{Materials and methods}

\section{JAK/STAT reporter gene}

A 441-bp genomic fragment in the enhancer of SOCS36E containing two potential STAT92E-binding sites was amplified by PCR, using five different sets of oligos: (1) CTGCAGGAAC CACTCAGAGTGCCTGCGTGT (PstI), GAATTCATACAAAA CTGTCTTAGGTGTTTA (EcoRI); (2) CTGCAGGAACCACT CAGAGTGCCTGCGTGT (PstI), CTGCAGATACAAAACT GTCTTAGGTGTTTA (PstI); (3) GAATTCGAACCACTCA GAGTGCCTGCGTGT (EcoRI), GAATTCATACAAAACTGT CTTAGGTGTTTA (EcoRI); (4) AGATCTGAACCACTCA GAGTGCCTGCGTGT (BglII), AGATCTATACAAAACTGT CTTAGGTGTTTA (BglII); (5) GCGGCCGCGAACCACTCAG AGTGCCTGCGTGT (NotI), GCGGCCGCATACAAAACTGT CTTAGGTGTTTA (NotI). Each amplified genomic fragment containing different restriction enzyme sites was sequentially subcloned into pP[UAST] (Phelps and Brand 1998). The genomic fragment amplified using the first set of oligos was subcloned into the PstI/EcoRI sites of pP[UAST] to generate 2XSTAT92E. The genomic fragment amplified using the second set of oligos was subcloned into the PstI site of 2XSTAT92E to generate 4XSTAT92E. The genomic fragment amplified using the third set of oligos was subcloned into the EcoRI site of 4XSTAT92E to generate 6XSTAT92E. The genomic fragment amplified using the fourth set of oligos was subcloned into the BglII site of 6XSTAT92E to generate 8XSTAT92E. Next, the hsp70 minimal promoter element was amplified from $\mathrm{pP}[\mathrm{UAST}]$ by PCR using oligos GCGGCCGCAGCGGAGACTCTAGCGAGCG (NotI) and CTCGAGAATTCCCTATTCAGAGTTCT (XhoI). This hsp70 minimal promoter was subcloned into the NotI/XhoI sites of 8XSTAT92E to generate 8XSTAT92E-hsp70. Again, the genomic fragment amplified using the fifth set of oligos was subcloned into the NotI site of 8XSTAT92E-hsp70 vector to generate 10XSTAT92E-hsp70. Finally, an XhoI/XbaI fragment containing the firefly luciferase gene from the pGL3-luciferase vector (Promega) was subcloned into the $\mathrm{Xhol} / \mathrm{XbaI}$ sites of 10XSTAT92E-hsp70 to generate 10XSTAT92E-luciferase.

To generate a reporter construct containing six STAT consensus sites, two pairs of oligos-(1) TTCTGGGAAACCGTTTA TACGCTGCGTTCGCGGAAACCGTTT ATACGCTGCGTTC TGGGAAACCGTTTATAC, AACGGTTTCCCAGAACGCAG 
CGTATAAACGGTTTCCGCGAACGCAGCGTATAAACGGTT TCCCAGAATGCA and (2) GCTGCGTTCGCGGAAACCGTT TA TACGCTGCGTTCTGGGAAACCGTTTATACGCTGCGT TCGCGGAA, AATTTTCCGCGAACGCAGCGTATAAACG GTTTCCCAGAACGCAGCGTATAAACGGTTTCCGCGAA CGCAGCGTATA-were annealed, respectively, and cloned together into pP[UAST] using PstI and EcoRI sites. Subsequently, an XhoI/XbaI fragment containing the firefly luciferase gene from the pGL3-luciferase vector (Promega) and the hsp70 minimal promoter were subcloned into the resulting vector using $\mathrm{XhoI} / \mathrm{XbaI}$ and NotI/XhoI sites, respectively, to generate the 6XSTAT-synthetic-luc construct.

\section{Cell lines}

The cell line that we used is a derivative of S2 cells and was originally a Peptidoglycan-responsive cell line. During the course of maintenance in our lab, however, we have noticed subtle morphological changes in these cells. Most importantly, they are no longer responsive to Peptidoglycan treatment. Thus, these cells have evolved to a new S2 cell derivative, and were thus referred to as "S2-NP."

\section{A cell-based RNAi screen}

A library of $\sim 21,300$ dsRNAs representing the Drosophila genome was aliquotted into 384-well plates ( $80 \mathrm{ng}$ dsRNA/well). For each well, $0.5 \mathrm{ng}$ 10XSTAT92E-luciferase, $10 \mathrm{ng}$ Act-Renilla, and $110 \mathrm{ng}$ pAc-PL (serving as carrier DNA) were mixed with $0.96 \mu \mathrm{L}$ Enhancer in $15 \mu \mathrm{L} \mathrm{EC}$ (Qiagen) and incubated at room temperature for $5 \mathrm{~min}$. Then $0.42 \mu \mathrm{L}$ of Effectene reagent were added and the mixture was immediately dispensed into each well containing dsRNA. After incubation at room temperature for $10 \mathrm{~min}, 40 \mu \mathrm{L}$ S2-NP cells $\left(10^{6}\right.$ cells $\left./ \mathrm{mL}\right)$ were dispensed into the well. Luciferase assays were performed $96 \mathrm{~h}$ after transfection using DualGlo reagents (Promega). For each well, the reporter activity, referred to as relative luciferase units (RLU), was calculated as the ratio of firefly luciferase to Renilla luciferase. For each plate, the mean and SD of RLU were calculated. dsRNAs that caused a RLU value to be either two SD or more below the mean or three SD or more above the mean were selected as candidate genes. The assay was conducted in duplicate to reduce the rate of false positives and to increase the reproducibility of individual candidates. For the secondary screen, 286 dsRNAs were resynthesized using the MegaScript kit (Ambion) and aliquotted into 384-well plates (80 ng dsRNA/ well). Transfection and luciferase assay were performed as described above. In experiments involving pol III-Renilla, the same amount of pol III-Renilla was used as with Act-Renilla. To assay candidate genes in cells stimulated with Upd, $20 \mathrm{pg}$ 10XSTAT92E-luciferase, 5 ng Act-Renilla, 105 ng pAc-PL, and $1 \mathrm{ng}$ Act-Upd were transfected to S2-NP cells together with 80 ng dsRNA. In experiments involving 6XSTAT-synthetic-luc, 2 ng 6XSTAT-synthetic-luc, 20 ng pol III-Renilla, 80 ng pAc-PL, and $20 \mathrm{ng}$ Act-Upd were transfected to S2-NP cells together with $80 \mathrm{ng}$ dsRNA per well. In all the above-mentioned experiments, luciferase assays were performed $96 \mathrm{~h}$ after transfection.

\section{Immunoprecipitation and Western blot analysis}

For analyzing Upd-induced STAT92E phosphorylation, an expression plasmid for HA-tagged STAT92E (Act-STAT92E-HA) was transfected into S2-NP cells together with dsRNA against $\mathrm{LacZ}$. Cells were split into two dishes $3.5 \mathrm{~d}$ after transfection. Half of the cells were cocultured with S2-NP cells transfected with Act-Upd $\sim 12 \mathrm{~h}$ prior to harvest (Harrison et al. 1998) and the other half remained untreated as control. Cell extracts were subjected to immunoprecipitation using anti-HA antibodies, and the immunoprecipitates were analyzed by immunoblotting analysis using anti-phospho-Tyr-STAT92E and anti-HA antibodies. The effect of RNAi knockdown of 29 potential positive regulators on STAT92E tyrosine phosphorylation was investigated by Western blot analysis. S2-NP cells were transfected with Act-STAT92E-HA together with dsRNA targeting each of the positive regulators. Four days after transfection, cells were cocultured with S2-NP cells transfected with Act-Upd for $\sim 12 \mathrm{~h}$ prior to harvest. The cell lysates were resolved by SDS-PAGE, transferred to PVDF membrane, and subjected to immunoblotting analysis using anti-phospho-Tyr-STAT92E antibody (Cell Signaling). The membrane was then stripped and reprobed with anti-HA antibody (Upstate) to detect STAT92E-HA as a loading control. To examine the effect of dsRNA-mediated knockdown of PTP61F on the phosphorylation status of Hop and STAT92E, Act-Myc-Hop or Act-STAT92E-HA was transfected into S2-NP cells together with dsRNAs against lacZ or PTP61F. Cells were harvested and cell lysates were immunoprecipitated with antiMyc or anti-HA antibodies, respectively. Immunoprecipitates were analyzed by immunoblotting using anti-phospho-Tyr or anti-phospho-Tyr-STAT92E antibodies, respectively. The membranes were stripped and reprobed with anti-Myc or anti-HA antibodies, respectively.

\section{Immunohistochemistry}

For image analysis, cells were bathed with various dsRNAs for $4 \mathrm{~d}$ and then transfected with Act-Upd or left untreated. Twenty-four hours after transfection, cells were fixed and standard immunohistochemistry was performed using an antibody against phospho-Tyr-STAT92E. DAPI staining was employed to visualize the nuclei. Accumulation of phosphorylated STAT92E in the nuclei was analyzed and images acquired under the confocal microscope.

\section{Fly stocks and genetic interaction}

Fly lines were maintained according to standard procedure. The following fly lines were used: $h o p^{C 11} / F M 7$ (Binari and Perrimon 1994), hop ${ }^{\text {Tum-1 }} / F M 7$ (a dominant temperature-sensitive allele) (Hanratty and Dearolf 1993), paired-Gal4 (Brand and Perrimon 1993), UAS-Upd (Harrison et al. 1998), UAS-Upd GMR-Gal4/ CyO (this study), UAS-PTP61F (this study), and UAS-PTP61F $D f(3) E D 4238 / T M 3$ (this study). Females carrying germline clones of hop ${ }^{C 111}$ were generated using the FLP-DFS technique (Chou and Perrimon 1996). Virgin females of the genotype hop ${ }^{C 111} F R T^{101} / F M 7$ were mated with males of the genotype OVO $^{D 1} F R T^{101} / Y ; F L P^{38}$. The resulting larvae were heat-shocked for $2 \mathrm{~h}$ at $37^{\circ} \mathrm{C}$. hop ${ }^{C 11} F R T^{101} / O V O^{D 1} F R T^{101}$ females were crossed with $F M 7 / Y$ males. For PTP61F genetic interaction studies, the eye phenotype of UAS-Upd GMR-Ga14/+ adult flies was compared to that of UAS-Upd GMR-Gal4/+; Df(3)ED4238/+

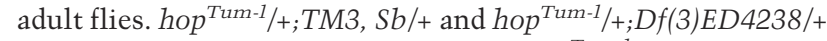
females were generated by crossing hop $p^{\text {Tum-1 }} / Y$ males with $D f(3) E D 4238 / T M 3, S b$ females, were maintained at $29^{\circ} \mathrm{C}$, and were scored for viability and the presence of melanotic tumors.

\section{Acknowledgments}

We thank Dr. Kent Nybakken for kindly providing the ActRenilla and pol III-Renilla plasmids. We thank members of the Drosophila RNAi Screening Center for reagents and technical assistance. We thank Ramanuj Dasgupta and Kent Nybakken 
for communicating data prior to publication. Special thanks go to Bernard Mathey-Provot, Sara Cherry, Ramanuj Dasgupta, Pamela Bradley, and Richard Binari for critically reading the manuscript. N.P. is a Howard Hughes Medical Institute investigator. G.-H.B. was supported by The Medical Foundation/ Charles A. King Trust post-doctoral fellowship. R.Z. is a Leukemia and Lymphoma Society fellow.

\section{Note added in proof}

Supplementary Table 1 lists the information on the dsRNAs. Please note that the results obtained with dsRNAs with potential off-target effects will need further validation with newly synthesized independent dsRNAs.

\section{References}

Andl, C.D., Mizushima, T., Oyama, K., Bowser, M., Nakagawa, H., and Rustgi, A.K. 2004. EGFR-induced cell migration is mediated predominantly by the JAK-STAT pathway in primary esophageal keratinocytes. Am. J. Physiol. Gastrointest. Liver Physiol. 287: G1227-G1237.

Aoki, N. and Matsuda, T. 2000. A cytosolic protein-tyrosine phosphatase PTP1B specifically dephosphorylates and deactivates prolactin-activated STAT5a and STAT5b. J. Biol. Chem. 275: 39718-39726.

Bach, E.A., Vincent, S., Zeidler, M.P., and Perrimon, N. 2003. A sensitized genetic screen to identify novel regulators and components of the Drosophila janus kinase/signal transducer and activator of transcription pathway. Genetics 165: 1149-1166.

Bai, J., Chiu, W., Wang, J., Tzeng, T., Perrimon, N., and Hsu, J. 2001. The cell adhesion molecule Echinoid defines a new pathway that antagonizes the Drosophila EGF receptor signaling pathway. Development 128: 591-601.

Binari, R. and Perrimon, N. 1994. Stripe-specific regulation of pair-rule genes by hopscotch, a putative Jak family tyrosine kinase in Drosophila. Genes \& Dev. 8: 300-312.

Boutros, M., Kiger, A.A., Armknecht, S., Kerr, K., Hild, M., Koch, B., Haas, S.A., Consortium, H.F., Paro, R., and Perrimon, N. 2004. Genome-wide RNAi analysis of growth and viability in Drosophila cells. Science 303: 832-835.

Brand, A.H. and Perrimon, N. 1993. Targeted gene expression as a means of altering cell fates and generating dominant phenotypes. Development 118: 401-415.

Bromberg, J.F. 2001. Activation of STAT proteins and growth control. Bioessays 23: 161-169.

Callus, B.A. and Mathey-Prevot, B. 2002. SOCS36E, a novel Drosophila SOCS protein, suppresses JAK/STAT and EGF-R signalling in the imaginal wing disc. Oncogene 21: 48124821.

Chen, X., Oh, S.W., Zheng, Z., Chen, H.W., Shin, H.H., and Hou, S.X. 2003. Cyclin D-Cdk4 and cyclin E-Cdk2 regulate the Jak/STAT signal transduction pathway in Drosophila. Dev. Cell 4: 179-190.

Chou, T.B. and Perrimon, N. 1996. The autosomal FLP-DFS technique for generating germline mosaics in Drosophila melanogaster. Genetics 144: 1673-1679.

Dasgupta, R., Kaykas, A., Moon, R.T., and Perrimon, N. 2005. Functional genomic analysis of the Wingless/Wnt signaling pathway. Science 308: 826-833.

Dearolf, C.R. 1998. Fruit fly 'leukemia.' Biochim. Biophys. Acta 1377: M13-M23.

Durbin, J.E., Hackenmiller, R., Simon, M.C., and Levy, D.E.
1996. Targeted disruption of the mouse Stat1 gene results in compromised innate immunity to viral disease. Cell 84: 443-450.

Hanratty, W.P. and Dearolf, C.R. 1993. The Drosophila Tumorous-lethal hematopoietic oncogene is a dominant mutation in the hopscotch locus. Mol. Gen. Genet. 238: 33-37.

Harrison, D.A., Binari, R., Nahreini, T.S., Gilman, M., and Perrimon, N. 1995. Activation of a Drosophila Janus kinase (JAK) causes hematopoietic neoplasia and developmental defects. $E M B O$ J. 14: 2857-2865.

Harrison, D.A., McCoon, P.E., Binari, R., Gilman, M., and Perrimon, N. 1998. Drosophila unpaired encodes a secreted protein that activates the JAK signaling pathway. Genes \& Dev. 12: 3252-3263.

Hild, M., Beckmann, B., Haas, S.A., Koch, B., Solovyev, V., Busold, C., Fellenberg, K., Boutros, M., Vingron, M., Sauer, F., et al. 2003. An integrated gene annotation and transcriptional profiling approach towards the full gene content of the Drosophila genome. Genome Biol. 5: R3.

Hou, X.S., Melnick, M.B., and Perrimon, N. 1996. Marelle acts downstream of the Drosophila HOP/JAK kinase and encodes a protein similar to the mammalian STATs. Cell 84: 411419.

Hou, S.X., Zheng, Z., Chen, X., and Perrimon, N. 2002. The Jak/STAT pathway in model organisms: Emerging roles in cell movement. Dev. Cell 3: 765-778.

Islam, R., Wei, S.Y., Chiu, W.H., Hortsch, M., and Hsu, J.C. 2003. Neuroglian activates Echinoid to antagonize the Drosophila EGF receptor signaling pathway. Development 130: 2051-2059.

Karsten, P., Hader, S., and Zeidler, M.P. 2002. Cloning and expression of Drosophila SOCS36E and its potential regulation by the JAK/STAT pathway. Mech. Dev. 117: 343-346.

Leaman, D.W., Pisharody, S., Flickinger, T.W., Commane, M.A., Schlessinger, J., Kerr, I.M., Levy, D.E., and Stark, G.R. 1996. Roles of JAKs in activation of STATs and stimulation of c-fos gene expression by epidermal growth factor. Mol. Cell. Biol. 16: 369-375.

McBride, K.M., McDonald, C., and Reich, N.C. 2000. Nuclear export signal located within the DNA-binding domain of the STAT1 transcription factor. EMBO I. 19: 6196-6206.

Meraz, M.A., White, J.M., Sheehan, K.C., Bach, E.A., Rodig, S.J., Dighe, A.S., Kaplan, D.H., Riley, J.K., Greenlund, A.C., Campbell, D., et al. 1996. Targeted disruption of the Stat 1 gene in mice reveals unexpected physiologic specificity in the JAK-STAT signaling pathway. Cell 84: 431-442.

Myers, M.P., Andersen, J.N., Cheng, A., Tremblay, M.L., Horvath, C.M., Parisien, J.P., Salmeen, A., Barford, D., and Tonks, N.K. 2001. TYK2 and JAK2 are substrates of proteintyrosine phosphatase 1B. J. Biol. Chem. 276: 47771-47774.

Neubauer, H., Cumano, A., Muller, M., Wu, H., Huffstadt, U., and Pfeffer, K. 1998. Jak2 deficiency defines an essential developmental checkpoint in definitive hematopoiesis. Cell 93: 397-409.

Nosaka, T., van Deursen, J.M., Tripp, R.A., Thierfelder, W.E., Witthuhn, B.A., McMickle, A.P., Doherty, P.C., Grosveld, G.C., and Ihle, J.N. 1995. Defective lymphoid development in mice lacking Jak3. Science 270: 800-802.

Parganas, E., Wang, D., Stravopodis, D., Topham, D.J., Marine, J.C., Teglund, S., Vanin, E.F., Bodner, S., Colamonici, O.R., van Deursen, J.M., et al. 1998. Jak2 is essential for signaling through a variety of cytokine receptors. Cell 93: 385-395.

Park, S.Y., Saijo, K., Takahashi, T., Osawa, M., Arase, H., Hirayama, N., Miyake, K., Nakauchi, H., Shirasawa, T., and Saito, T. 1995. Developmental defects of lymphoid cells in Jak3 kinase-deficient mice. Immunity 3: 771-782. 
Baeg et al.

Phelps, C.B. and Brand, A.H. 1998. Ectopic gene expression in Drosophila using GAL4 system. Methods 14: 367-379.

Quelle, F.W., Thierfelder, W., Witthuhn, B.A., Tang, B., Cohen, S., and Ihle, J.N. 1995. Phosphorylation and activation of the DNA binding activity of purified Statl by the Janus proteintyrosine kinases and the epidermal growth factor receptor. J. Biol. Chem. 270: 20775-20780.

Rodig, S.J., Meraz, M.A., White, J.M., Lampe, P.A., Riley, J.K., Arthur, C.D., King, K.L., Sheehan, K.C., Yin, L., Pennica, D., et al. 1998. Disruption of the Jak1 gene demonstrates obligatory and nonredundant roles of the Jaks in cytokine-induced biologic responses. Cell 93: 373-383.

Sekimoto, T., Imamoto, N., Nakajima, K., Hirano, T., and Yoneda, Y. 1997. Extracellular signal-dependent nuclear import of Stat 1 is mediated by nuclear pore-targeting complex formation with NPI-1, but not Rch1. EMBO J. 16: 7067-7077.

Shuai, K. and Liu, B. 2003. Regulation of JAK-STAT signalling in the immune system. Nat. Rev. Immunol. 3: 900-911.

Tao, W., Zhang, S., Turenchalk, G.S., Stewart, R.A., St John, M.A., Chen, W., and Xu, T. 1999. Human homologue of the Drosophila melanogaster lats tumour suppressor modulates CDC2 activity. Nat. Genet. 21: 177-181.

Thomis, D.C., Gurniak, C.B., Tivol, E., Sharpe, A.H., and Berg, L.J. 1995. Defects in B lymphocyte maturation and T lymphocyte activation in mice lacking Jak3. Science 270: 794797.

Velazquez, L., Fellous, M., Stark, G.R., and Pellegrini, S. 1992. A protein tyrosine kinase in the interferon $\alpha / \beta$ signaling pathway. Cell 70: 313-322.

Vogel, B.E. and Hedgecock, E.M. 2001. Hemicentin, a conserved extracellular member of the immunoglobulin superfamily, organizes epithelial and other cell attachments into oriented line-shaped junctions. Development 128: 883-894.

Yan, R., Luo, H., Darnell Jr., J.E., and Dearolf, C.R. 1996. A JAK-STAT pathway regulates wing vein formation in Drosophila. Proc. Natl. Acad. Sci. 93: 5842-5847.

$\mathrm{Yu}, \mathrm{H}$. and Jove, R. 2004. The STATs of cancer-New molecular targets come of age. Nat. Rev. Cancer 4: 97-105. 
Addendum

Genes \& Development 19: 1861-1870 (2005)

Genome-wide RNAi analysis of JAK/STAT signaling components in Drosophila

Gyeong-Hun Baeg, Rui Zhou, and Norbert Perrimon

Recently it was shown that long double-stranded RNAs (dsRNAs) can lead to "off-target effects" (OTE) in Drosophila cells (Kulkarni et al. 2006; Ma et al. 2006). We therefore generated one or two additional independent dsRNAs for each of the 121 candidate genes of the JAK/STAT signaling pathway that we initially reported (Baeg et al. 2005). Each of the newly generated dsRNAs was designed to be free of 19 base pairs (bp) or longer overlap with other genes. We retested these new dsRNAs in parallel with the original dsRNAs identified from the screen and found that 111 original dsRNAs scored, and among them, 50 could be further confirmed by one or two independent dsRNAs (Table 1). Of interest, we note that 17 of the original dsRNAs that were devoid of any 19-bp homology with other genes failed to be confirmed by additional dsRNAs. This finding suggests that other OTE rules that we have not been able to identify (such as interference with miRNA function through potential seed regions found in small interfering RNAs [siRNAs] [Lewis et al. 2003]) may also lead to false positives in large-scale screens in Drosophila cells. Alternatively, it is possible that knockdown efficiency varies among different long dsRNAs. In addition, nine of the dsRNAs in the initial 121 positives that, based on our in silico analysis, were predicted to have off-target sequences targeting 15 or more other genes could be confirmed with a second or third dsRNA. Taken together, our data strongly support the recommendation made by Echeverri et al. (2006) that testing of two or more independent dsRNAs should be performed, and will help minimize the risk of reporting false positives in RNA interference (RNAi)-based assays. In conclusion, cell-based assays and RNAi, when well controlled, constitute a valid approach for identification of genes potentially involved in a given biological process, but more detailed biochemical and genetic analyses will be necessary to validate these candidate genes.

Table 1. List of genes that were confirmed by two or three independent dsRNAs in the JAK/STAT assay

\begin{tabular}{|c|c|c|c|c|c|}
\hline \multicolumn{3}{|c|}{ Negative regulators } & \multicolumn{3}{|c|}{ Positive regulators } \\
\hline Amplicon & Gene & Fold change & Amplicon & Gene & Fold change \\
\hline DRSC 11325 & ash1 & 3.43 & DRSC03504 & $\operatorname{cdc} 2$ & 0.40 \\
\hline DRSC32654 & ash1 & 3.81 & DRSC30705 & $\operatorname{cdc} 2$ & 0.39 \\
\hline DRSC32655 & ash1 & 2.64 & DRSC30706 & $\operatorname{cdc} 2$ & 0.38 \\
\hline DRSC19337 & Bap60 & 1.47 & DRSC17794 & CG11700 & 0.27 \\
\hline DRSC32656 & Bap60 & 1.70 & DRSC31545 & CG11700 & 0.22 \\
\hline DRSC32657 & Bap60 & 1.53 & DRSC08254 & CG12104 & 0.65 \\
\hline DRSC 11330 & brm & 1.89 & DRSC32318 & CG12104 & 0.67 \\
\hline DRSC30901 & brm & 1.32 & DRSC32317 & CG12104 & 0.55 \\
\hline DRSC03287 & Cas & 1.43 & DRSC15283 & CG17836 & 0.24 \\
\hline DRSC32658 & Cas & 1.48 & DRSC32680 & CG17836 & 0.29 \\
\hline DRSC32659 & Cas & 1.76 & DRSC32681 & CG17836 & 0.29 \\
\hline DRSC04085 & CG10955 & 1.43 & DRSC18386 & CG32767 & 0.30 \\
\hline DRSC30727 & CG10955 & 1.55 & DRSC32396 & CG32767 & 0.54 \\
\hline DRSC30728 & CG10955 & 1.81 & DRSC13053 & CG3563 & 0.54 \\
\hline DRSC09878 & CG12310 & 2.22 & DRSC30936 & CG3563 & 0.73 \\
\hline DRSC25358 & CG12310 & 1.48 & DRSC10516 & CG5546 & 0.44 \\
\hline DRSC04191 & CG13550 & 2.44 & DRSC30863 & CG5546 & 0.34 \\
\hline DRSC31776 & CG13550 & 1.37 & DRSC30862 & CG5546 & 0.49 \\
\hline DRSC31777 & CG13550 & 1.50 & DRSC 10563 & CG5971 & 0.78 \\
\hline DRSC00447 & CG15432 & 1.48 & DRSC32714 & CG5971 & 0.63 \\
\hline DRSC32675 & CG15432 & 1.25 & DRSC19969 & CG5988 & 0.26 \\
\hline DRSC06127 & CG30089 & 3.36 & DRSC32715 & CG5988 & 0.23 \\
\hline DRSC32689 & CG30089 & 1.27 & DRSC32716 & CG5988 & 0.35 \\
\hline DRSC10977 & CG32365 & 1.74 & DRSC18427 & CG8636 & 0.35 \\
\hline DRSC32700 & CG32365 & 1.29 & DRSC32087 & CG8636 & 0.41 \\
\hline DRSC11697 & CG32428 & 2.80 & DRSC32088 & CG8636 & 0.55 \\
\hline DRSC32701 & CG32428 & 1.24 & DRSC19583 & dome & 0.17 \\
\hline DRSC06562 & CG33455 & 2.01 & DRSC32731 & dome & 0.16 \\
\hline DRSC32709 & CG33455 & 1.47 & DRSC32732 & dome & 0.13 \\
\hline
\end{tabular}


Table 1. (continued)

\begin{tabular}{|c|c|c|c|c|c|}
\hline \multicolumn{3}{|c|}{ Negative regulators } & \multicolumn{3}{|c|}{ Positive regulators } \\
\hline Amplicon & Gene & Fold change & Amplicon & Gene & Fold change \\
\hline DRSC32710 & CG33455 & 1.38 & DRSC16704 & Hmgcr & 0.34 \\
\hline DRSC04360 & CG3363 & 1.37 & DRSC31628 & Hmgcr & 0.77 \\
\hline DRSC32064 & CG3363 & 1.90 & DRSC20340 & hop & 0.23 \\
\hline DRSC18349 & CG4136 & 3.83 & DRSC32739 & hop & 0.25 \\
\hline DRSC32406 & CG4136 & 3.27 & DRSC32740 & hop & 0.23 \\
\hline DRSC32407 & CG4136 & 3.59 & DRSC00708 & lilli & 0.56 \\
\hline DRSC10635 & CG6434, CG5585 & 1.51 & DRSC32745 & lilli & 0.49 \\
\hline DRSC32720 & CG6434 & 1.24 & DRSC32746 & lilli & 0.65 \\
\hline DRSC11848 & CG7752 & 1.53 & DRSC 11251 & Pdp1 & 0.58 \\
\hline DRSC31702 & CG7752 & 1.29 & DRSC32517 & Pdp1 & 0.77 \\
\hline DRSC31701 & CG7752 & 1.48 & DRSC11285 & Snap & 0.54 \\
\hline DRSC20132 & CG8949 & 1.89 & DRSC31261 & Snap & 0.20 \\
\hline DRSC32727 & CG8949 & 1.44 & DRSC16870 & Stat92E & 0.17 \\
\hline DRSC32728 & CG8949 & 1.43 & DRSC32773 & Stat92E & 0.23 \\
\hline DRSC04096 & enok & 2.51 & DRSC32774 & Stat92E & 0.27 \\
\hline DRSC32735 & enok & 1.81 & DRSC00843 & ush & 0.14 \\
\hline DRSC32736 & enok & 2.38 & DRSC32226 & ush & 0.42 \\
\hline DRSC16651 & jumu & 1.59 & DRSC32227 & ush & 0.34 \\
\hline DRSC32741 & jumu & 2.02 & & & \\
\hline DRSC32742 & jumu & 1.81 & & & \\
\hline DRSC04696 & ken & 4.83 & & & \\
\hline DRSC31748 & ken & 1.69 & & & \\
\hline DRSC06948 & lolal & 1.76 & & & \\
\hline DRSC32751 & lolal & 1.64 & & & \\
\hline DRSC32752 & lolal & 1.78 & & & \\
\hline DRSC 15378 & mor & 1.35 & & & \\
\hline DRSC32754 & mor & 1.30 & & & \\
\hline DRSC14209 & Nup98 & 1.38 & & & \\
\hline DRSC31803 & Nup98 & 1.29 & & & \\
\hline DRSC11874 & Pitslre & 1.55 & & & \\
\hline DRSC31971 & Pitslre & 1.56 & & & \\
\hline DRSC08683 & Ptp61F & 4.39 & & & \\
\hline DRSC32761 & Ptp61F & 4.52 & & & \\
\hline DRSC32762 & Ptp61F & 4.01 & & & \\
\hline DRSC17034 & puc & 1.84 & & & \\
\hline DRSC31024 & puc & 1.34 & & & \\
\hline DRSC02455 & Socs $36 E$ & 5.46 & & & \\
\hline DRSC30658 & Socs $36 E$ & 2.32 & & & \\
\hline DRSC30659 & Socs $36 E$ & 5.54 & & & \\
\hline DRSC16211 & Ssdp & 1.46 & & & \\
\hline DRSC31311 & Ssdp & 1.29 & & & \\
\hline DRSC31310 & Ssdp & 1.51 & & & \\
\hline DRSC11309 & Trn & 1.53 & & & \\
\hline DRSC32778 & Trn & 1.22 & & & \\
\hline DRSC17089 & $\operatorname{trx}$ & 4.90 & & & \\
\hline DRSC32779 & $\operatorname{trx}$ & 4.20 & & & \\
\hline DRSC32780 & $\operatorname{trx}$ & 5.87 & & & \\
\hline DRSC20381 & unc-4 & 1.99 & & & \\
\hline DRSC32781 & unc- 4 & 1.61 & & & \\
\hline DRSC03641 & $\mathrm{zf} 30 \mathrm{C}$ & 1.55 & & & \\
\hline DRSC32783 & $\mathrm{zf} 30 \mathrm{C}$ & 1.83 & & & \\
\hline DRSC32784 & $\mathrm{zf} 30 \mathrm{C}$ & 1.77 & & & \\
\hline
\end{tabular}

The average reporter activity from multiple control samples treated with lac $Z$ dsRNA was set as 1 and those from samples treated with test dsRNAs were calculated accordingly. Results shown were the average values from three independent experiments conducted in duplicate. The cut-off values are 1.2 and 0.8 for negative and positive regulators, respectively. The original amplicons are shaded. 


\section{References}

Baeg, G.H., Zhou, R., and Perrimon, N. 2005. Genome-wide RNAi analysis of JAK/STAT signaling components in Drosophila. Genes \& Dev. 19: 1861-1870.

Echeverri, C.J., Beachy, P.A., Baum, B., Boutros, M., Buchholz, F., Chanda, S.K., Downward, J., Ellenberg, J., Fraser, A.G., Hacohen, N., et al. 2006. Minimizing the risk of reporting false positives in large-scale RNAi screens. Nat. Methods 3: 777-779.

Kulkarni, M.M., Booker, M., Silver, S.J., Friedman, A., Hong, P., Perrimon, N., and Mathey-Prevot, B. 2006. Evidence of offtarget effects associated with long dsRNAs in Drosophila melanogaster cell-based assays. Nat. Methods 3: 833-838.

Lewis, B.P., Shih, I.H., Jones-Rhoades, M.W., Bartel, D.P., and Burge, C.B. 2003. Prediction of mammalian microRNA targets. Cell 115: 787-798.

Ma, Y., Creanga, A., Lum, L., and Beachy, P.A. 2006. Prevalence of off-target effects in Drosophila RNA interference screens. Nature 443: 359-363. 


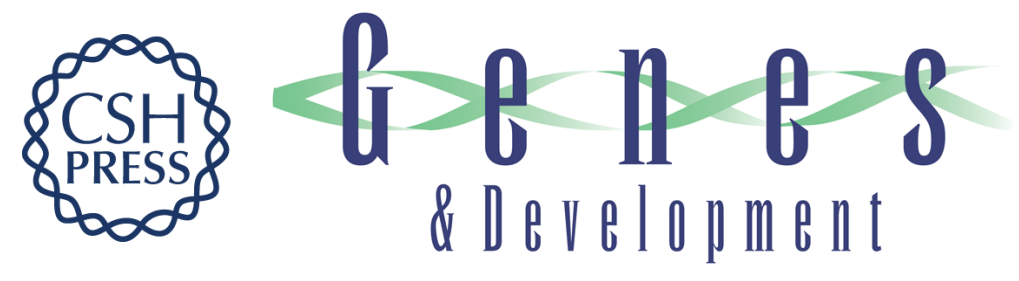

\section{Genome-wide RNAi analysis of JAK/STAT signaling components in Drosophila}

Gyeong-Hun Baeg, Rui Zhou and Norbert Perrimon

Genes Dev. 2005, 19:

Access the most recent version at doi:10.1101/gad.1320705

\section{Supplemental http://genesdev.cshlp.org/content/suppl/2005/07/29/gad.1320705.DC1 Material}

Related Content

References

\section{License}

Email Alerting

Service

\section{Addendum}

Genes Dev. April , 2007 21: 875-877

This article cites 41 articles, 19 of which can be accessed free at: http://genesdev.cshlp.org/content/19/16/1861.full.html\#ref-list-1

Articles cited in:

http://genesdev.cshlp.org/content/19/16/1861.full.html\#related-urls

Receive free email alerts when new articles cite this article - sign up in the box at the top right corner of the article or click here.

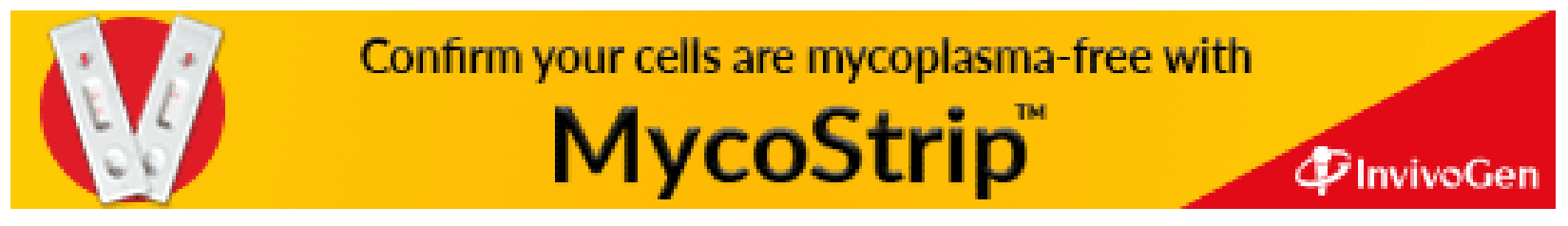

